\title{
Yield and Yield Components of Durum Wheat as Influenced by Humic Acid, Zinc and Iron Application
}

\author{
Idris O. Al-Mahdi ${ }^{1}$, Sami Sh. EL-Tabbakh ${ }^{1}$, Ali I. Nawar ${ }^{1}$ and Mahmoud H. Abd-Elmoneim ${ }^{1}$
}

\begin{abstract}
A two-year field study was carried out at the Agricultural Experiment Station, Alexandria University in $2017 / 2018$ and $2018 / 2019$ winter seasons to investigate the effect of foliar spraying of humic acid (HA) (0 and 2.4 $\mathrm{kg} / \mathrm{ha})$ and both $\mathrm{Fe}$ and $\mathrm{Zn}$ micronutrients $(0,480$ and 960 $\mathrm{g} / \mathrm{ha}$ of $\mathrm{FeSO}_{4}$ and/or $\mathrm{ZnSO}_{4}$, respectively) on three durum wheat cultivars (Casino, Bani Sweif6 and Sohag3). The experiment was laid out in split-plot design with three replications, where combinations of $\mathrm{HA}$ levels and cultivars occupied the main plots, while combinations of $\mathrm{Fe}$ and $\mathrm{Zn}$ levels were randomly allocated to the sub plots.

Bani Sweif6 was superior to the other two cultivars for all studied grain yield and yield components except for 100-grain weight. Application of humic acid increased grain yield of Bani Sweif6 and Sohag3, but negatively affected that of Casino. Application of $480 \mathrm{~g} / \mathrm{ha}$ of both $\mathrm{FeSO}_{4}$ and $\mathrm{ZnSO}_{4}$ gave the highest values with $\mathrm{HA}$ application in Bani Sweif6 and Sohag3, and without HA application for Casino. It is recommended to spray Bani Sweif6 and Sohag3 with $2.4 \mathrm{~kg} / \mathrm{ha} \mathrm{HA}$ and $480 \mathrm{~g} / \mathrm{ha}$ of both $\mathrm{Fe}$ and $\mathrm{Zn}$, while more studies are needed to determine the suitable level of HA spraying for Casino cultivar.
\end{abstract}

Keywords: Durum wheat, humic acid, iron, zinc, grain yield, yield components.

\section{INTRODUCTION}

Durum wheat (Triticum durum) is of significance as a food crop used to make traditional foods. It is widely adapted and it is mostly grown in semi-arid regions. World production of durum wheats is increasing for its use in producing healthy, low-glycemic-index foods (Kadkol and Sissons, 2016). Increasing wheat productivity in Egypt, as a representative to arid or semi-arid regions, is confronted with many problems including water shortage in non-irrigated areas, poor soil fertility in marginal areas in addition to soil salinity or sodicity. Hence, application of soil ammendments and spraying with essential micronutrients is a necessity to ensure a profitable level of productivity for wheat in these areas, beside cultivation of the suitable wheat cultivar.

Humic acid (HA) is an organic substance that has beneficial effects on wheat growth and productivity such as increasing photosynthetic metabolism, water retention and bioavailability of micronutrients throughout the growing period of wheat plants
(Mackowiak et al., 2001, Delfine et al., 2005). Antoun et al. (2010) found that application of humic acid increased grain and straw yields, and 1000-grain weight. Khan et al. (2010) reported that application of $3.0 \mathrm{~kg} / \mathrm{ha}$ humic acid increased grain yield of wheat by $24 \%$ compared to the control. Similarly, Doorodian et al. (2015) found that application of humic acid at the rate of $8 \mathrm{~L} / \mathrm{ha}$ significantly increased number of tillers and spikes/plant, number of grains/spike, 1000-grain weight and grain yield. Moreover, Yasin and EL-sobky (2017) reported that wheat plants treated with $4 \mathrm{~kg} / \mathrm{fed}$ humic acid had higher number of spikes $/ \mathrm{m}^{2}$, number of grains/spike, 1000-kernel weight, biological, straw and grain yields compared to untreated control.

Micronutrients, including iron and zinc, are essential elements for improving plant growth and mediate several biochemical processes in plants. The role of iron and zinc in promoting wheat plants growth and productivity has been established by several researchers. Hussain et al. (2005) reported that spraying wheat plants with micronutrients including $\mathrm{Zn}$ and $\mathrm{Fe}$ increased grain and biological yields, but did not affect harvest index. Gomaa et al. (2015) found that application of zinc and iron significantly increased number of spikes $/ \mathrm{m}^{2}$, number of grains/spike, 1000grain weight, grain, straw and biological yields and harvest index. Similar results were reported by Ramzan et al. (2020) and Jalal et al. (2020).

The present investigation was carried out to study the effect of humic acid application combined with foliar spraying with zinc and iron on yield and yield components of two local and one introduced durum wheat cultivars.

\section{MATERIAL AND METHODS}

Two field experiments were carried out at the Agricultural Research Station, Alexandria University, Egypt, during the two successive wheat growing winter seasons of 2017/2018 and 2018/2019 to investigate of the effect of spraying with humic acid and micronutrients $(\mathrm{Fe}, \mathrm{Zn})$ and their interactions on growth and productivity of three durum wheat cultivars, two local (Bani Sweif6 and Sohag3) and one introduced from Libya (Casino). 
Table 1. Soil Physical and chemical properties as an average of the two seasons

\begin{tabular}{|c|c|c|c|c|c|}
\hline Soil character & Average & $\begin{array}{c}\text { Chemical } \\
\text { properties }\end{array}$ & Average & $\begin{array}{c}\text { Chemical } \\
\text { properties }\end{array}$ & Average \\
\hline Physical & & Av. N\% & 0.01 & $\mathrm{Na}^{+}$meq/ L & 20.21 \\
\hline properties & & Av. P meq/ L & 9.60 & $\mathrm{Zn}^{+2} \mathrm{PPm}$ & 2.5 \\
\hline Sand $\%$ & 62.5 & Av. K meq/ L & 0.84 & $\mathrm{Fe}^{+2} \mathrm{PPm}$ & 2.9 \\
\hline Silt \% & 20.0 & O.M. \% & 0.52 & $\mathrm{Cl}^{-} \mathrm{meq} / \mathrm{L}$ & 15.00 \\
\hline Clay \% & 17.5 & $\mathrm{pH}$ & 8.36 & $\mathrm{CO}_{3}^{-2} \mathrm{meq} / \mathrm{L}$ & 2.40 \\
\hline \multirow{3}{*}{ Texture } & \multirow{3}{*}{ Sand loam } & Ec $(\mathrm{dS} / \mathrm{m})$ & 2.23 & $\mathrm{HCO}_{3}{ }^{-} \mathrm{meq} / \mathrm{L}$ & 4.00 \\
\hline & & $\mathrm{Ca}^{+2} \mathrm{meq} / \mathrm{L}$ & 7.50 & $\mathrm{CaCO}_{3}(\%)$ & 9.86 \\
\hline & & $\mathrm{Mg}^{+2} \mathrm{meq} / \mathrm{L}$ & 4.00 & SAR & 5.96 \\
\hline
\end{tabular}

The experiments were sown after maize in the two seasons. Soil physical and chemical characteristics in both seasons were determined using soil samples collected before sowing at each experimental site from 0-30 $\mathrm{cm}$ depth for analysis according to Page et al. (1982) and Klute (1986) The full analysis of soil samples are presented in Table (1)

Each experiment included four factors, i.e. three durum wheat cultivars, two levels of humic acid (0 and $2.4 \mathrm{~kg} / \mathrm{ha}$ ) sprayed after 30 days from sowing (DAS). Three levels of Fe (0, 480 and $960 \mathrm{~g} / \mathrm{ha}$ as iron sulphate) sprayed in two equal doses after 30 and 45 DAS and three levels of $\mathrm{Zn}(0,480$ and $960 \mathrm{~g} / \mathrm{ha}$ as zinc sulphate) sprayed in two equal doses after 30 and 45 DAS. Sowing date was 18 November in the two seasons. Seeding rate in the two seasons was $120 \mathrm{~kg} / \mathrm{ha}$ for the three cultivars. Phosphorus was applied at the rate of 38 $\mathrm{kg} / \mathrm{ha}$, as calcium monophosphate $\left(15.5 \% \mathrm{P}_{2} \mathrm{O}_{5}\right)$ during seed bed preparation. Nitrogen was applied at the rate of $192 \mathrm{~kg} / \mathrm{ha}$, as ammonium nitrate $(33.5 \% \mathrm{~N})$ in three doses, $48 \mathrm{~kg} \mathrm{~N} / \mathrm{ha}$ during land preparation and the remaining quantity was applied in two equal doses, each of $72 \mathrm{~kg} \mathrm{~N} / \mathrm{ha}$, applied just before the second and third irrigations after sowing. Potassium, as potassium sulphate $\left(48 \% \mathrm{~K}_{2} \mathrm{O}\right)$, was applied at the rate of $57.6 \mathrm{~kg}$ $\mathrm{K}_{2} \mathrm{O} / \mathrm{ha}$ at 30 DAS. Cultural practices, such as pests control and irrigation were carried out as recommended for durum wheat production in Alexandria region.

The experimental design in the two seasons was a split plot with three replications. Combinations of wheat cultivars and humic acid levels occupied the main plots, whereas combinations of $\mathrm{Fe}$ and $\mathrm{Zn}$ levels were allocated to the sub plots. Sub plots area was $3.6 \mathrm{~m}^{2}$ comprising four rows, each $3 \mathrm{~m}$ long and $0.3 \mathrm{~m}$ wide.

At harvest, yield and yield components were recorded. These included: number of spikes $/ \mathrm{m}^{2}$ $\left(\mathrm{NS} / \mathrm{m}^{2}\right)$, number of grains/spike (NGS), 100 grain weight (HGW) (in grams), biological yield (BY) (ton/ha), grain yield (GY) (ton/ha).
Data were statistically analyzed according to Gomez and Gomez (1984) using SAS ver 9.1 (2002). Least significant differences values at 0.05 level of probability were used to compare the differences between treatments means. Quadratic regression analysis and equations were performed using Curve Expert, ver. 1.34 (Hyams, 2005).

\section{RESULTS AND DISCUSSION}

\section{Results}

Analysis of variance (Table 2) indicated that all yield and yield components were significantly affected by the four-factor interaction, in the two seasons, except number of grains/spike which was significantly influenced by cultivars in the two seasons, $\mathrm{H}^{*} \mathrm{~V}$ in the first season only, zinc levels in the two seasons, $\mathrm{Fe} * \mathrm{H} * \mathrm{~V}$ and $\mathrm{Zn} * \mathrm{H} * \mathrm{~V}$ in the second season only.

Means of the levels of studied factors (Table3) indicated that Bani Sweif 6 gave higher values than the other two cultivars for the studied traits except for 100grain weight, in the two seasons, where Sohag3 exhibited heavier grain weight. Spraying with $2.4 \mathrm{~kg} / \mathrm{ha}$ humic acid gave, generally, higher values for all studied yield attributes compared to control. Moreover, spraying with the intermediate level ( $480 \mathrm{~g} / \mathrm{ha})$ of each of $\mathrm{Fe}$ and $\mathrm{Zn}$ gave higher values for all yield and yield components compared to control or the highest level of each $(960 \mathrm{~g} / \mathrm{ha})$. However, the response of the studied durum wheat cultivars differed with application of humic acid, Fe and $\mathrm{Zn}$ levels as indicated by the significance of the various interactions between studied factors.

Means for number of spikes $/ \mathrm{m}^{2}$ as affected by the four-factor interaction are presented in (Table 4). The data revealed that the three cultivars recorded the significantly highest values for that character with spraying with humic acid at $2.4 \mathrm{~kg} / \mathrm{ha}$, and the intermediate level (480 g/ha) of both Fe and $\mathrm{Zn}$. 
Table 2. Mean squares for No. of spikes $/ \mathrm{m}^{2}$, No. of grains/spike, 100- grain weight biological yield, grain yield and as affected by wheat cultivar, humic acid, iron and zinc levels and their interactions two seasons

\begin{tabular}{|c|c|c|c|c|c|c|c|c|c|c|c|}
\hline \multirow[t]{2}{*}{ S.O.V } & \multirow[t]{2}{*}{ d.f } & \multicolumn{2}{|c|}{$\mathrm{NS} / \mathrm{m}^{2}$} & \multicolumn{2}{|c|}{ NG /S } & \multicolumn{2}{|c|}{ HGW } & \multicolumn{2}{|c|}{ BY } & \multicolumn{2}{|c|}{ GY } \\
\hline & & $\mathbf{S}_{1}$ & $\mathbf{S}_{2}$ & $\mathbf{S}_{1}$ & $\mathbf{S}_{2}$ & $\mathbf{S}_{1}$ & $\mathbf{S}_{2}$ & $\mathbf{S}_{1}$ & $\mathbf{S}_{2}$ & $\mathbf{S}_{1}$ & $\mathbf{S}_{2}$ \\
\hline Cultivar (C) & 2 & $* *$ & $* *$ & $* *$ & $* *$ & $* *$ & $* *$ & $* *$ & $* *$ & $* *$ & $* *$ \\
\hline $\begin{array}{l}\text { Humic } \\
\operatorname{acid}(H)\end{array}$ & 1 & $* *$ & $* *$ & N.S & N.S & $* *$ & $* *$ & N.S & $* *$ & $* *$ & $* *$ \\
\hline $\mathrm{H}^{*} \mathrm{~V}$ & 2 & $* *$ & N.S & $* *$ & N.S & $* *$ & $* *$ & N.S & $* *$ & $* *$ & $* *$ \\
\hline $\mathrm{Ea}$ & 10 & 52.48 & 122.70 & 32.31 & 59.27 & 0.016 & 0.005 & 0.455 & 0.594 & 0.036 & 0.052 \\
\hline $\operatorname{Iron}(\mathrm{Fe})$ & 2 & $* *$ & $* *$ & N.S & N.S & $* *$ & $* *$ & $* *$ & $* *$ & $* *$ & $* *$ \\
\hline $\operatorname{Zinc}(\mathrm{Zn})$ & 2 & $* *$ & $* *$ & $*$ & $*$ & $* *$ & $* *$ & $* *$ & $* *$ & $* *$ & $* *$ \\
\hline $\mathrm{Zn} * \mathrm{~V}$ & 4 & N.S & $* *$ & N.S & N.S & $* *$ & $* *$ & $* *$ & N.S & $* *$ & N.S \\
\hline $\mathrm{Fe}^{*} \mathrm{~V}$ & 4 & $* *$ & $* *$ & N.S & N.S & $* *$ & $* *$ & $* *$ & $* *$ & $* *$ & $* *$ \\
\hline $\mathrm{Fe}^{*} \mathrm{H}$ & 2 & $* *$ & $* *$ & N.S & N.S & $* *$ & $* *$ & $* *$ & N.S & $* *$ & $* *$ \\
\hline $\mathrm{Zn} * \mathrm{H}$ & 2 & $* *$ & $* *$ & N.S & N.S & $* *$ & $* *$ & N.S & N.S & $* *$ & $* *$ \\
\hline $\mathrm{Zn} * \mathrm{Fe}$ & 4 & $* *$ & $* *$ & N.S & N.S & $* *$ & $* *$ & $* *$ & $* *$ & $* *$ & $* *$ \\
\hline $\mathrm{Fe}^{*} \mathrm{H}^{*} \mathrm{~V}$ & 4 & $* *$ & $* *$ & N.S & * & $* *$ & $* *$ & N.S & $* *$ & $* *$ & $* *$ \\
\hline $\mathrm{Zn} * \mathrm{H} * \mathrm{~V}$ & 4 & $* *$ & $* *$ & N.S & $* *$ & $* *$ & $* *$ & $* *$ & $* *$ & $* *$ & $* *$ \\
\hline $\mathrm{Zn} * \mathrm{Fe}^{*} \mathrm{H}$ & 4 & $* *$ & $* *$ & N.S & N.S & $* *$ & $* *$ & $* *$ & $* *$ & $* *$ & $* *$ \\
\hline $\mathrm{Zn} * \mathrm{Fe}^{*} \mathrm{~V}$ & 8 & $* *$ & $* *$ & N.S & N.S & $* *$ & $* *$ & $* *$ & $* *$ & $* *$ & $* *$ \\
\hline $\mathrm{Zn} * \mathrm{Fe}^{*} \mathrm{H}^{*} \mathrm{~V}$ & 8 & $* *$ & $* *$ & N.S & N.S & $* *$ & $* *$ & $* *$ & $* *$ & $* *$ & $* *$ \\
\hline $\mathrm{Eb}$ & 96 & 64.69 & 121.2 & 29.96 & 30.23 & 0.012 & 0.009 & 0.425 & 0.428 & 0.033 & 0.038 \\
\hline
\end{tabular}

*,** significant at 0.05 and 0.01 probability levels, respectively.

S1: $2017 / 2018$ season

S2: 2018/2019 season

Table 3. Means for No. of spikes $/ \mathrm{m}^{2}$, No. of grains/spike, 100-grain weight biological yield, grain yield, as affected by wheat cultivar, humic acid, iron and zinc levels in the two seasons

\begin{tabular}{|c|c|c|c|c|c|c|c|c|c|c|}
\hline \multirow{2}{*}{ Treatments } & \multicolumn{2}{|c|}{$\mathbf{N S} / \mathrm{m}^{2}$} & \multicolumn{2}{|c|}{ NG /S } & \multicolumn{2}{|c|}{ HGW(g) } & \multicolumn{2}{|c|}{ BY(ton/ha) } & \multicolumn{2}{|c|}{ GY(ton/ha) } \\
\hline & $\mathbf{S}_{\mathbf{1}}$ & $\mathbf{S}_{\mathbf{2}}$ & $\mathbf{S}_{\mathbf{1}}$ & $\mathbf{S}_{2}$ & $\mathbf{S}_{1}$ & $\mathbf{S}_{2}$ & $\mathbf{S}_{1}$ & $\mathbf{S}_{2}$ & $\mathbf{S}_{\mathbf{1}}$ & $\overline{\mathbf{S}_{2}}$ \\
\hline \multicolumn{11}{|c|}{ Cultivar } \\
\hline Casino & $316.01 \mathrm{c}$ & $322.42 c$ & $49.26 \mathrm{~b}$ & $55.33 \mathrm{~b}$ & $5.08 b$ & $5.28 b$ & $7.04 \mathrm{c}$ & $9.89 \mathrm{~b}$ & $1.87 \mathrm{c}$ & $2.72 \mathrm{c}$ \\
\hline Bani sweif6 & $351.53 \mathrm{a}$ & $357.13 a$ & $53.69 \mathrm{a}$ & $59.96 \mathrm{a}$ & $5.02 b$ & $5.16 \mathrm{c}$ & $9.55 \mathrm{a}$ & $11.54 \mathrm{a}$ & $2.94 \mathrm{a}$ & $3.99 \mathrm{a}$ \\
\hline Sohag3 & $341.35 b$ & $347.38 b$ & $47.11 \mathrm{~b}$ & $52.19 \mathrm{~b}$ & $5.27 \mathrm{a}$ & $5.42 \mathrm{a}$ & $7.50 \mathrm{~b}$ & $9.99 \mathrm{~b}$ & $2.39 \mathrm{~b}$ & $3.27 b$ \\
\hline L.S.D. 0.05 & 3.10 & 4.74 & 2.43 & 3.30 & 0.055 & 0.032 & 0.289 & 0.330 & 0.081 & 0.098 \\
\hline \multicolumn{11}{|c|}{ Humic acid levels kg/ha } \\
\hline Control & $322.51 b$ & $329.9 \mathrm{~b}$ & $49.72 \mathrm{a}$ & $56.30 \mathrm{a}$ & $4.93 b$ & $5.11 \mathrm{~b}$ & $7.97 \mathrm{a}$ & $10.07 \mathrm{~b}$ & $2.23 b$ & $3.26 \mathrm{~b}$ \\
\hline 2.4 & $350.08 \mathrm{a}$ & $354.72 \mathrm{a}$ & $50.32 \mathrm{a}$ & $55.33 \mathrm{a}$ & $5.32 \mathrm{a}$ & $5.46 \mathrm{a}$ & $8.09 \mathrm{a}$ & $10.88 \mathrm{a}$ & $2.57 \mathrm{a}$ & $3.39 \mathrm{a}$ \\
\hline L.S.D. 0.05 & 2.53 & 3.87 & N.S & N.S & 0.044 & 0.026 & N.S & 0.269 & 0.066 & 0.080 \\
\hline \multicolumn{11}{|c|}{ Iron levels g/ha } \\
\hline Control & $331.96 b$ & $338.59 b$ & $50.33 a$ & $56.17 \mathrm{a}$ & $5.01 \mathrm{~b}$ & $5.19 \mathrm{~b}$ & $8.13 b$ & $10.19 \mathrm{~b}$ & $2.39 \mathrm{~b}$ & $3.19 b$ \\
\hline 480 & $357.61 \mathrm{a}$ & $362.22 \mathrm{a}$ & $49.68 \mathrm{a}$ & $55.63 \mathrm{a}$ & $5.39 \mathrm{a}$ & $5.54 \mathrm{a}$ & $9.02 \mathrm{a}$ & $11.47 \mathrm{a}$ & $2.80 \mathrm{a}$ & $3.79 \mathrm{a}$ \\
\hline 960 & $319.33 c$ & $326.13 c$ & $50.06 \mathrm{a}$ & $55.65 \mathrm{a}$ & $4.97 \mathrm{c}$ & $5.13 \mathrm{c}$ & $6.94 c$ & $9.77 \mathrm{c}$ & $2.02 \mathrm{c}$ & $3.00 \mathrm{c}$ \\
\hline L.S.D. 0.05 & 3.07 & 4.20 & N.S & N.S & 0.042 & 0.037 & 0.249 & 0.250 & 0.069 & 0.074 \\
\hline \multicolumn{11}{|c|}{ Zinc levels g/ha } \\
\hline Control & $334.16 b$ & $340.37 b$ & $49.43 b$ & $56.32 \mathrm{a}$ & $5.03 b$ & $5.22 b$ & $7.60 \mathrm{~b}$ & $9.91 b$ & $2.28 \mathrm{~b}$ & $3.15 b$ \\
\hline 480 & $354.11 \mathrm{a}$ & $358.63 \mathrm{a}$ & $51.69 \mathrm{a}$ & $56.82 \mathrm{a}$ & $5.38 \mathrm{a}$ & $5.49 \mathrm{a}$ & $9.46 \mathrm{a}$ & $11.47 \mathrm{a}$ & $2.89 \mathrm{a}$ & $3.73 \mathrm{a}$ \\
\hline 960 & $320.63 c$ & $327.94 \mathrm{c}$ & $48.94 b$ & $54.32 \mathrm{~b}$ & $4.97 \mathrm{c}$ & $5.16 \mathrm{c}$ & $7.03 \mathrm{c}$ & $10.05 b$ & $2.03 \mathrm{c}$ & $3.10 \mathrm{~b}$ \\
\hline L.S.D. 0.05 & 3.07 & 4.20 & 2.09 & 2.10 & 0.04 & 0.04 & 0.25 & 0.25 & 0.07 & 0.07 \\
\hline
\end{tabular}


Spraying of the highest level of $\mathrm{Fe}$ or $\mathrm{Zn}$, or both, decreased significantly number of spikes $/ \mathrm{m}^{2}$ even with application of humic acid. However, in Casino cultivar, application of humic acid improved the values of that character at highest levels of $\mathrm{Fe}$ or $\mathrm{Zn}$, whereas, in Bani Sweif6 and Sohag3, the lowest values were obtained with spraying of humic acid and $\mathrm{Fe}$ and $\mathrm{Zn}$ at $960 \mathrm{~g} / \mathrm{ha}$. The same trend of data was observed for 100-grain weight, with the exception that humic acid application improved that character at highest $\mathrm{Fe}$ and $\mathrm{Zn}$ levels compared to non-spraying of humic acid (Table 4) in all cultivars.

Table 4. Means of No. of spikes $/ \mathrm{m}^{2}\left(\mathrm{NS} / \mathrm{m}^{2}\right)$ and 100 - grain weight (HWG)as affected by wheat cultivar * humic acid $\mathrm{kg} / \mathrm{ha}$ * iron $\mathrm{g} / \mathrm{ha}$ * zinc $\mathrm{g} / \mathrm{ha}$ interactionat in the two seasons

\begin{tabular}{|c|c|c|c|c|c|c|c|}
\hline \multirow[t]{2}{*}{ Cultivars } & \multirow{2}{*}{$\begin{array}{c}\text { Humic } \\
\text { acid } \\
\mathrm{kg} / \mathrm{ha}\end{array}$} & \multirow{2}{*}{$\begin{array}{l}\text { Iron } \\
\text { g/ha }\end{array}$} & \multirow{2}{*}{$\begin{array}{l}\text { Zinc } \\
\text { g/ha }\end{array}$} & \multicolumn{2}{|c|}{$\mathbf{N S} / \mathbf{m}^{2}$} & \multicolumn{2}{|c|}{ HGW (g) } \\
\hline & & & & $\mathbf{S}_{1}$ & $\mathbf{S}_{2}$ & $\mathbf{S}_{1}$ & $\mathbf{S}_{2}$ \\
\hline Casino & 0 & 0 & 0 & 253.6 & 260.3 & 4.23 & 4.45 \\
\hline Casino & 0 & 0 & 480 & 326.0 & 332.3 & 5.04 & 5.23 \\
\hline Casino & 0 & 0 & 960 & 307.0 & 322.6 & 4.98 & 5.20 \\
\hline Casino & 0 & 480 & 0 & 343.6 & 350.3 & 5.26 & 5.47 \\
\hline Casino & 0 & 480 & 480 & 354.0 & 362.0 & 5.53 & 5.69 \\
\hline Casino & 0 & 480 & 960 & 300.3 & 311.6 & 5.24 & 5.42 \\
\hline Casino & 0 & 960 & 0 & 300.0 & 314.0 & 4.95 & 5.13 \\
\hline Casino & 0 & 960 & 480 & 263.0 & 268.6 & 5.12 & 5.22 \\
\hline Casino & 0 & 960 & 960 & 244.3 & 253.0 & 4.62 & 4.94 \\
\hline Casino & 2.4 & 0 & 0 & 331.0 & 327.0 & 4.40 & 4.53 \\
\hline Casino & 2.4 & 0 & 480 & 342.0 & 348.3 & 5.17 & 5.42 \\
\hline Casino & 2.4 & 0 & 960 & 319.0 & 329.0 & 5.15 & 5.55 \\
\hline Casino & 2.4 & 480 & 0 & 340.0 & 348.3 & 5.13 & 5.41 \\
\hline Casino & 2.4 & 480 & 480 & 423.3 & 401.0 & 6.15 & 6.24 \\
\hline Casino & 2.4 & 480 & 960 & 318.0 & 324.6 & 5.18 & 5.33 \\
\hline Casino & 2.4 & 960 & 0 & 311.3 & 319.3 & 5.22 & 5.34 \\
\hline Casino & 2.4 & 960 & 480 & 311.6 & 321.0 & 5.17 & 5.28 \\
\hline Casino & 2.4 & 960 & 960 & 300.0 & 310.0 & 4.96 & 5.25 \\
\hline Bani sweif6 & 0 & 0 & 0 & 326.6 & 336.0 & 5.11 & 5.32 \\
\hline Bani sweif6 & 0 & 0 & 480 & 336.6 & 345.0 & 5.16 & 5.22 \\
\hline Bani sweif6 & 0 & 0 & 960 & 323.6 & 331.3 & 4.53 & 4.67 \\
\hline Bani sweif6 & 0 & 480 & 0 & 338.0 & 343.3 & 5.15 & 5.37 \\
\hline Bani sweif6 & 0 & 480 & 480 & 362.2 & 369.3 & 5.24 & 5.20 \\
\hline Bani sweif6 & 0 & 480 & 960 & 344.0 & 347.3 & 4.35 & 4.48 \\
\hline Bani sweif6 & 0 & 960 & 0 & 326.3 & 334.3 & 4.56 & 4.96 \\
\hline Bani sweif6 & 0 & 960 & 480 & 347.0 & 351.6 & 4.37 & 4.44 \\
\hline Bani sweif6 & 0 & 960 & 960 & 337.3 & 342.0 & 4.28 & 4.35 \\
\hline Bani sweif6 & 2.4 & 0 & 0 & 363.6 & 370.0 & 5.20 & 5.38 \\
\hline Bani sweif6 & 2.4 & 0 & 480 & 376.3 & 379.0 & 5.17 & 5.28 \\
\hline Bani sweif6 & 2.4 & 0 & 960 & 355.3 & 361.0 & 5.14 & 5.39 \\
\hline Bani sweif6 & 2.4 & 480 & 0 & 389.0 & 390.0 & 5.20 & 5.36 \\
\hline Bani sweif6 & 2.4 & 480 & 480 & 442.6 & 446.6 & 6.44 & 6.43 \\
\hline Bani sweif6 & 2.4 & 480 & 960 & 337.0 & 342.3 & 5.27 & 5.43 \\
\hline Bani sweif6 & 2.4 & 960 & 0 & 356.3 & 360.0 & 5.15 & 5.33 \\
\hline Bani sweif6 & 2.4 & 960 & 480 & 346.0 & 351.6 & 5.45 & 5.54 \\
\hline Bani sweif6 & 2.4 & 960 & 960 & 319.0 & 327.3 & 4.75 & 4.77 \\
\hline
\end{tabular}


Continue table 4.

\begin{tabular}{|c|c|c|c|c|c|c|c|}
\hline \multirow[t]{2}{*}{ Cultivars } & \multirow[t]{2}{*}{$\begin{array}{c}\text { Humic } \\
\text { acid } \\
\mathrm{kg} / \mathrm{ha}\end{array}$} & \multirow[t]{2}{*}{$\begin{array}{l}\text { Iron } \\
\text { g/ha }\end{array}$} & \multirow[t]{2}{*}{$\begin{array}{l}\text { Zinc } \\
\text { g/ha }\end{array}$} & \multicolumn{2}{|c|}{ N S } & \multicolumn{2}{|c|}{ HGW (g) } \\
\hline & & & & $\mathbf{S}_{1}$ & $\mathbf{S}_{2}$ & $\mathbf{S}_{1}$ & $\mathbf{S}_{2}$ \\
\hline Sohag3 & 0 & 0 & 0 & 316.0 & 322.6 & 4.90 & 4.97 \\
\hline Sohag3 & 0 & 0 & 480 & 330.6 & 338.0 & 5.00 & 5.25 \\
\hline Sohag3 & 0 & 0 & 960 & 312.6 & 319.6 & 5.05 & 5.23 \\
\hline Sohag3 & 0 & 480 & 0 & 331.3 & 338.3 & 5.01 & 5.32 \\
\hline Sohag3 & 0 & 480 & 480 & 354.6 & 360.0 & 5.58 & 5.80 \\
\hline Sohag3 & 0 & 480 & 960 & 337.6 & 344.6 & 5.18 & 5.34 \\
\hline Sohag3 & 0 & 960 & 0 & 318.6 & 327.0 & 4.97 & 5.12 \\
\hline Sohag3 & 0 & 960 & 480 & 344.0 & 346.6 & 5.11 & 5.24 \\
\hline Sohag3 & 0 & 960 & 960 & 328.0 & 335.0 & 4.74 & 5.12 \\
\hline Sohag3 & 2.4 & 0 & 0 & 349.3 & 356.6 & 5.11 & 5.30 \\
\hline Sohag3 & 2.4 & 0 & 480 & 357.0 & 362.6 & 5.61 & 5.70 \\
\hline Sohag3 & 2.4 & 0 & 960 & 348.6 & 353.0 & 5.37 & 5.50 \\
\hline Sohag3 & 2.4 & 480 & 0 & 373.6 & 378.6 & 5.69 & 5.78 \\
\hline Sohag3 & 2.4 & 480 & 480 & 416.0 & 424.3 & 6.12 & 6.17 \\
\hline Sohag3 & 2.4 & 480 & 960 & 331.0 & 337.0 & 5.41 & 5.51 \\
\hline Sohag3 & 2.4 & 960 & 0 & 346.3 & 350.3 & 5.35 & 5.46 \\
\hline Sohag3 & 2.4 & 960 & 480 & 340.3 & 347.0 & 5.46 & 5.52 \\
\hline Sohag3 & 2.4 & 960 & 960 & 308.3 & 311.3 & 5.28 & 5.41 \\
\hline \multicolumn{4}{|c|}{ L.S.D. 0.05} & 39.08 & 17.83 & 0.18 & 0.15 \\
\hline
\end{tabular}

Table 5. Means of number of grains/spike as affected by cultivars * humic acid interaction in the first season $\left(\mathbf{S}_{1}\right)$

\begin{tabular}{ccc}
\hline Cultivars & $\begin{array}{c}\text { Humic } \\
\text { acid kg/ha }\end{array}$ & $\begin{array}{c}\text { No. of grains/spike } \\
\mathbf{S}_{\mathbf{1}}\end{array}$ \\
\hline Casino & 0 & 51.296 \\
Casino & 2.4 & 47.222 \\
Bani sweif6 & 0 & 53.814 \\
Bani sweif6 & 2.4 & 53.555 \\
Sohag3 & 0 & 44.037 \\
Sohag3 & 2.4 & 50.185 \\
\hline L.S.D. 0.05 & 3.447 \\
\hline
\end{tabular}

With regard to number of grains/spike, the cultivar* humic acid interaction in the first season (Table 5) revealed that Casino cultivars responded significantly and negatively, Bani Sweif6 showed no significant response, while Sohage3 responed significantly and positively to spray application of $2.4 \mathrm{~kg} / \mathrm{ha}$ humic acid. Moreover the three factor interaction, cultivar*humic acid*iron concentration in the second season (Table 6) showed that Casino cultivars gave the highest value for that character with $480 \mathrm{~g} \mathrm{Fe} / \mathrm{ha}$ without humic acid application, while Bani Sweif6 gave the highest means with $480 \mathrm{~g} \mathrm{Fe} / \mathrm{ha}$ without humic acid, application of humic acid only, and application of humic acid $+960 \mathrm{~g}$
$\mathrm{Fe} / \mathrm{ha}$. On the other hand, Sohag3 gave the highest value with control and spraying with $960 \mathrm{~g} \mathrm{Fe} / \mathrm{ha}$ only. In addition, the three factor interaction, cultivar*humic acid*zinc (Table 7), revealed that Casino responded positively to 480 and $960 \mathrm{~g} \mathrm{Zn} /$ ha without humic acid, Bani Sweif6 gave the highest value at $480 \mathrm{~g} \mathrm{Zn} / \mathrm{ha}+2.4$ $\mathrm{kg} / \mathrm{ha}$ humic acid, and Sohag3 had the highest number of grains/spike when sprayed with $480 \mathrm{~g} \mathrm{Zn} / \mathrm{ha}$ with and without application of humic acid in the second season.

Concerning biological yield (BY) (Table 8), the four-factor interaction indicated that Casino gave the highest values in absence of humic acid with different combinations of levels of $\mathrm{Fe}$ and or $\mathrm{Zn}$. However, application of the highest level of both micronutrients reduced biological yield significantly in the two seasons. On the other hand, Bani Sweif6 and sohag3 cultivars gave the highest biological yield, in the two seasons, with spraying of humic acid and both micronutrients at the intermediate level of $480 \mathrm{~g} / \mathrm{ha}$. Moreover, biological yield of Bani sweif6 cultivar decreased significantly with application of the two micronutrients at $960 \mathrm{~g} / \mathrm{ha}$ with or without humic acid application.

With regard to grain yield (GY), means of the fourfactor interaction presented in (Table 8) revealed that grain yield followed the same trend of biological yield for Bani Sweif6 and Sohag3, whereas Casino, in the 
first season showed a positive response for humic acid application and $\mathrm{Zn}$ application at $480 \mathrm{~g} / \mathrm{ha}$ and which was at par with application of $\mathrm{Zn}$ and $\mathrm{Fe}$ at $480 \mathrm{~g} / \mathrm{ha}$ (2.92 and $2.76 \mathrm{t} / \mathrm{ha}$, respectively). However, in the second season, humic acid application had a negative effect on grain yield for both Bani Sweif6 and Sohag3, where the highest grain yield values were recorded for spraying with humic acid at $2.4 \mathrm{~kg} / \mathrm{ha}$ and spraying both $\mathrm{Fe}$ and $\mathrm{Zn}$ at the rate of $480 \mathrm{~g} / \mathrm{ha}$ (4.72 and 5.67 and 3.78 and $4.76 \mathrm{t} / \mathrm{ha}$ for the first and second season, respectively).

Table 6. Means of No. of grains/spike as affected by Cultivars * humic acid * iron interaction in the second season $\left(\mathbf{S}_{2}\right)$

\begin{tabular}{|c|c|c|c|c|}
\hline Cultivars & Humic acid kg/ha & Iron $\mathrm{g} / \mathrm{ha}$ & No. of grains/spike & $\mathbf{S}_{2}$ \\
\hline casino & 0 & 0 & 55.777 & \\
\hline casino & 0 & 480 & 60.666 & \\
\hline casino & 0 & 960 & 52.000 & \\
\hline casino & 2.4 & 0 & 53.888 & \\
\hline casino & 2.4 & 480 & 53.444 & \\
\hline casino & 2.4 & 960 & 56.222 & \\
\hline Bani sweif6 & 0 & 0 & 58.555 & \\
\hline Bani sweif6 & 0 & 480 & 60.888 & \\
\hline Bani sweif6 & 0 & 960 & 59.444 & \\
\hline Bani sweif6 & 2.4 & 0 & 61.444 & \\
\hline Bani sweif6 & 2.4 & 480 & 58.666 & \\
\hline Bani sweif6 & 2.4 & 960 & 60.777 & \\
\hline Sohag3 & 0 & 0 & 54.777 & \\
\hline Sohag3 & 0 & 480 & 49.666 & \\
\hline Sohag3 & 0 & 960 & 54.888 & \\
\hline Sohag3 & 2.4 & 0 & 52.555 & \\
\hline Sohag3 & 2.4 & 480 & 50.444 & \\
\hline Sohag3 & 2.4 & 960 & 50.555 & \\
\hline & L.S.D. 0.05 & & 1.053 & \\
\hline
\end{tabular}

Table 7. Means of No. of grains/spike as affected by Cultivars * humic acid * zinc interaction in the second season $\left(\mathbf{S}_{2}\right)$

\begin{tabular}{|c|c|c|c|c|}
\hline Cultivars & Humic acid kg/ha & g/ha & No. of grains/spike & $\mathbf{S}_{2}$ \\
\hline Casino & 0 & 0 & 52.888 & \\
\hline Casino & 0 & 480 & 57.777 & \\
\hline Casino & 0 & 960 & 57.777 & \\
\hline Casino & 2.4 & 0 & 55.444 & \\
\hline Casino & 2.4 & 480 & 53.000 & \\
\hline Casino & 2.4 & 960 & 55.111 & \\
\hline Bani sweif6 & 0 & 0 & 61.333 & \\
\hline Bani sweif6 & 0 & 480 & 56.000 & \\
\hline Bani sweif6 & 0 & 960 & 61.555 & \\
\hline Bani sweif6 & 2.4 & 0 & 56.777 & \\
\hline Bani sweif6 & 2.4 & 480 & 63.000 & \\
\hline Bani sweif6 & 2.4 & 960 & 61.111 & \\
\hline Sohag3 & 0 & 0 & 51.777 & \\
\hline Sohag3 & 0 & 480 & 55.333 & \\
\hline Sohag3 & 0 & 960 & 52.222 & \\
\hline Sohag3 & 2.4 & 0 & 47.666 & \\
\hline Sohag3 & 2.4 & 480 & 55.777 & \\
\hline Sohag3 & 2.4 & 960 & 50.111 & \\
\hline & L.S.D. 0.05 & & 1.053 & \\
\hline
\end{tabular}


Table 8. Means of Biological yield and Grain yield as affected by Cultivars * humic acid * iron *zinc interaction in the two seasons

\begin{tabular}{|c|c|c|c|c|c|c|c|}
\hline \multirow[t]{2}{*}{ Cultivars } & \multirow{2}{*}{$\begin{array}{l}\text { Humic acid } \\
\mathrm{kg} / \mathrm{ha}\end{array}$} & \multirow[t]{2}{*}{ Iron g/ha } & \multirow[t]{2}{*}{ Zinc g/ha } & \multicolumn{2}{|c|}{ BY (t /ha) } & \multicolumn{2}{|c|}{ GY (t /ha) } \\
\hline & & & & $\mathbf{S}_{1}$ & $\mathbf{S}_{r}$ & $\mathbf{S}_{1}$ & $\mathbf{S}_{r}$ \\
\hline Casino & 0 & 0 & 0 & 7.04 & 7.70 & 1.78 & 2.03 \\
\hline Casino & 0 & 0 & 480 & 8.57 & 11.86 & 2.22 & 3.12 \\
\hline Casino & 0 & 0 & 960 & 8.25 & 11.76 & 2.03 & 3.05 \\
\hline Casino & 0 & 480 & 0 & 4.90 & 10.32 & 1.27 & 2.78 \\
\hline Casino & 0 & 480 & 480 & 9.52 & 12.24 & 2.68 & 3.59 \\
\hline Casino & 0 & 480 & 960 & 8.34 & 12.64 & 2.06 & 3.30 \\
\hline Casino & 0 & 960 & 0 & 8.39 & 12.2 & 2.20 & 3.24 \\
\hline Casino & 0 & 960 & 480 & 4.81 & 12.12 & 1.32 & 3.49 \\
\hline Casino & 0 & 960 & 960 & 3.65 & 8.16 & 0.88 & 2.10 \\
\hline Casino & 2.4 & 0 & 0 & 5.56 & 10.19 & 1.46 & 2.82 \\
\hline Casino & 2.4 & 0 & 480 & 10.38 & 9.28 & 2.92 & 2.83 \\
\hline Casino & 2.4 & 0 & 960 & 5.83 & 10.24 & 1.54 & 2.81 \\
\hline Casino & 2.4 & 480 & 0 & 8.40 & 8.63 & 2.36 & 2.49 \\
\hline Casino & 2.4 & 480 & 480 & 9.75 & 11.06 & 2.76 & 3.23 \\
\hline Casino & 2.4 & 480 & 960 & 6.42 & 8.63 & 1.73 & 2.45 \\
\hline Casino & 2.4 & 960 & 0 & 5.90 & 7.09 & 1.63 & 2.03 \\
\hline Casino & 2.4 & 960 & 480 & 6.17 & 7.86 & 1.64 & 2.13 \\
\hline Casino & 2.4 & 960 & 960 & 4.96 & 6.36 & 1.25 & 1.67 \\
\hline Bani sweif6 & 0 & 0 & 0 & 8.58 & 9.56 & 2.44 & 2.93 \\
\hline Bani sweif6 & 0 & 0 & 480 & 10.96 & 12.12 & 3.19 & 3.99 \\
\hline Bani sweif6 & 0 & 0 & 960 & 7.79 & 12.13 & 2.13 & 3.86 \\
\hline Bani sweif6 & 0 & 480 & 0 & 10.45 & 12.25 & 2.86 & 3.89 \\
\hline Bani sweif6 & 0 & 480 & 480 & 11.95 & 14.49 & 3.51 & 4.87 \\
\hline Bani sweif6 & 0 & 480 & 960 & 8.38 & 11.85 & 2.30 & 3.87 \\
\hline Bani sweif6 & 0 & 960 & 0 & 7.70 & 12.08 & 2.27 & 3.87 \\
\hline Bani sweif6 & 0 & 960 & 480 & 12.42 & 12.66 & 3.57 & 4.02 \\
\hline Bani sweif6 & 0 & 960 & 960 & 5.70 & 8.48 & 1.62 & 2.65 \\
\hline Bani sweif6 & 2.4 & 0 & 0 & 8.25 & 9.94 & 2.64 & 3.69 \\
\hline Bani sweif6 & 2.4 & 0 & 480 & 9.90 & 10.94 & 3.26 & 4.13 \\
\hline Bani sweif6 & 2.4 & 0 & 960 & 9.07 & 9.87 & 2.74 & 3.49 \\
\hline Bani sweif6 & 2.4 & 480 & 0 & 8.67 & 10.46 & 3.55 & 4.47 \\
\hline Bani sweif6 & 2.4 & 480 & 480 & 13.97 & 14.58 & 4.72 & 5.67 \\
\hline Bani sweif6 & 2.4 & 480 & 960 & 11.89 & 14.07 & 3.93 & 5.28 \\
\hline Bani sweif6 & 2.4 & 960 & 0 & 10.19 & 11.88 & 3.21 & 4.22 \\
\hline Bani sweif6 & 2.4 & 960 & 480 & 9.47 & 10.79 & 3.04 & 3.79 \\
\hline Bani sweif6 & 2.4 & 960 & 960 & 6.69 & 9.66 & 2.00 & 3.19 \\
\hline
\end{tabular}


Continue table 8 .

\begin{tabular}{lccccccc}
\hline Cultivars & $\begin{array}{c}\text { Humic acid } \\
\mathbf{k g} / \mathbf{h a}\end{array}$ & Iron $\mathbf{g} / \mathbf{h a}$ & Zinc g/ha & \multicolumn{2}{c}{$\mathbf{B Y}$ (t /ha) } & \multicolumn{2}{c}{ GY (t/ha) } \\
\cline { 5 - 7 } & & & & $\mathbf{S}_{\mathbf{1}}$ & $\mathbf{S}_{\mathbf{2}}$ & $\mathbf{S}_{\mathbf{1}}$ & $\mathbf{S}_{\mathbf{2}}$ \\
\hline Sohag3 & 0 & 0 & 0 & 6.50 & 7.07 & 1.91 & 2.16 \\
Sohag3 & 0 & 0 & 480 & 9.03 & 10.98 & 2.75 & 3.50 \\
Sohag3 & 0 & 0 & 960 & 8.31 & 10.76 & 2.48 & 3.35 \\
Sohag3 & 0 & 480 & 0 & 5.37 & 8.20 & 1.66 & 2.59 \\
Sohag3 & 0 & 480 & 480 & 10.17 & 13.20 & 3.13 & 4.18 \\
Sohag3 & 0 & 480 & 960 & 8.72 & 12.48 & 2.49 & 3.70 \\
Sohag3 & 0 & 960 & 0 & 9.44 & 12.20 & 2.67 & 3.56 \\
Sohag3 & 0 & 960 & 480 & 5.65 & 7.63 & 1.73 & 2.43 \\
Sohag3 & 0 & 960 & 960 & 4.70 & 7.04 & 1.33 & 205 \\
Sohag3 & 2.4 & 0 & 0 & 5.89 & 8.87 & 1.95 & 2.95 \\
Sohag3 & 2.4 & 0 & 480 & 10.49 & 11.40 & 3.59 & 4.02 \\
Sohag3 & 2.4 & 0 & 960 & 6.12 & 8.80 & 2.08 & 2.91 \\
Sohag3 & 2.4 & 480 & 0 & 9.34 & 10.08 & 3.33 & 3.79 \\
Sohag3 & 2.4 & 480 & 480 & 9.81 & 11.91 & 3.78 & 4.76 \\
Sohag3 & 2.4 & 480 & 960 & 6.31 & 9.39 & 2.29 & 3.34 \\
Sohag3 & 2.4 & 960 & 0 & 6.27 & 9.87 & 2.00 & 3.26 \\
Sohag3 & 2.4 & 960 & 480 & 7.32 & 11.38 & 2.33 & 3.57 \\
Sohag3 & 2.4 & 960 & 960 & 5.58 & 8.71 & 1.69 & 2.75 \\
\hline
\end{tabular}

Quadratic regression for grain yield as influenced by Fe levels and humic acid application for the different cultivars (Fig 1 a to c) revealed that grain yield increased with application of Fe up to 420.86, 458.9 and $378.71 \mathrm{~g} / \mathrm{ha}$ without humic acid application, and up $466.32,471.92$ and $431.48 \mathrm{~g} / \mathrm{ha}$ with application of 2.4 $\mathrm{kg} / \mathrm{ha}$ humic acid for Casino, Bani Sweif6 and Sohag3 cultivars, respectively, then decreased with higher application of Fe. Similar trend of results were found for $\mathrm{Zn}$ application (Fig 2 a to c) where grain yield increased with application of $\mathrm{Zn}$ levels up to 486.42, 442.99 and $517.94 \mathrm{~g} / \mathrm{ha}$ without humic acid application, and up to 432.77, 440.8 and $435.59 \mathrm{~g} / \mathrm{ha}$ with application of 2.4 $\mathrm{kg} / \mathrm{ha}$ humic acid for Casino, Bani Sweif6 and Sohag3 cultivars, respectively, then decreased with higher levels of $\mathrm{Zn}$.

\section{Discussion}

Increasing durum wheat productivity, as in all other field crops, require a critical balance of essential macroand micronutrients. Soils with high $\mathrm{pH}$ values limit the availability of micronutrients as $\left(\mathrm{Fe}^{+2}\right.$ and $\left.\mathrm{Zn}^{+2}\right)$ to plants (Table 1). Hence, it is recommended to spray $\mathrm{Fe}$ and $\mathrm{Zn}$ directly to plants for increasing their availability.
These two micronutrients play important roles in wheat growth and productivity since they are involved in several biochemical processes such as chlorophyll production and photosynthesis (Broadley et al., 2007; Fageria, 2009), affect the capacity for water uptake and transport (Kasim, 2007 and Disante et al. ,2010) and protein synthesis (Hansch and Mendel, 2009, Li et al. ,2012 and Finatto et al. ,2015).

Spraying with adequate concentrations of $\mathrm{Fe}$ and $\mathrm{Zn}$ micronutrients lead to enhancement of plant development and productivity. In the present study, spraying with both micronutrients at $480 \mathrm{~g} / \mathrm{ha}$ of each, increased grain yield and yield components of the studed durum wheat cultivars. Several researchers reported that spraying with $\mathrm{Fe}$ and or $\mathrm{Zn}$ increased wheat grain yield (Habib,2009 and 2012, Armin et al. 2014 and Jalal et al. 2020), 1000-grain weight (Pahlavan-Red and Pessarakli, 2009 and Monjezi et al. ,2013) number of tillers $/ \mathrm{m}^{2}$ (Nadim et al. ,2012 and Hassanein et al. 2019), number of grains per spike (Mekkei and EL-Haggan, 2014 and Ramzan et al. ,2020) and biological yield (Arif et al. ,2017 and Khaksar and Lack, 2019). 
The application of a higher dosage of $\mathrm{Fe}$ and $\mathrm{Zn}$ (960 g/ha of each) resulted in a negative impact on grain yield and yield components, and that may be attributed to several reasons including imbalance of the nutritive status of the wheat plant (Fageria, 2009), disrupting photosynthesis processes through inhibition of chlorophyll synthesis (Sandallio et al. ,2001) or through increasing oxidative injury which lead to disturbing metablic pathways affecting plant growth and development (Posmyk and Kontek, 2009). However, cultivars showed differential response to higher levels of $\mathrm{Fe}$ and $\mathrm{Zn}$, where Casino showed higher tolerance to $960 \mathrm{~g} / \mathrm{ha}$ of both micronutrients compared to Bani Sweif6 and Sohag3. Kabir et al. (2016) reported differential tolerance of wheat cultivars to excess iron. The ability of a wheat plant to tolerate excess $\mathrm{Fe}$ or $\mathrm{Zn}$ levels may be due to protective mechanisms established by the plant such as sequestration of excess micronutrients into the vacuoles (Tsonev and Lidon, 2012) or the presence of antioxidant enzymes defense mechanisms that play a vital role in alleviating the damage induced by heavy metal stress Li et al. ,2012, Kumar et al. ,2014 and Wu et al. ,2014).
The overall effect of humic acid (HA) application showed a positive response, significant or insignificant, to spraying of $2.4 \mathrm{~kg} / \mathrm{ha}$ HA compared to the control. Several researchers reported significant positive effects of HA on grain yield (Antoun et al., 2010, Khan et al. ,2010 and Manzoor et al. ,2014), 1000-grain weight (Knapowski et al. ,2015 and AL-Erwy et al. ,2016), number of tiller plant (Yasin and EL-Sobky,2017 and Baqir and Zeboo, 2019) biological yield (Kandil et al. ,2016 and Dincsoy and Sonmez, 2019) and number of grains/spike (Doroodian et al. ,2015 and Yassin and ELSobky 2017) of wheat plants. However, durum wheat cultivars showed differential response to application of HA (Fig 1 and 2, a, b and c) where both cultivars Bani Sweif6 and Sohag3 showed a positive response to HA application compared to control with regard to grain yield, while Casino cultivar revealed a negative response to HA spraying. That may be attributed to the differences in genetic make up of cultivars which may influence their response to the applied dose of HA. Several studies have reported positive, negative and no effects of HA application to wheat in relation to employed cultivars (Mackowiak et al. ,2001, Delfine et al. ,2005, Jones et al. ,2007, Lodhi et al. ,2013. and Radwan et al,2014).

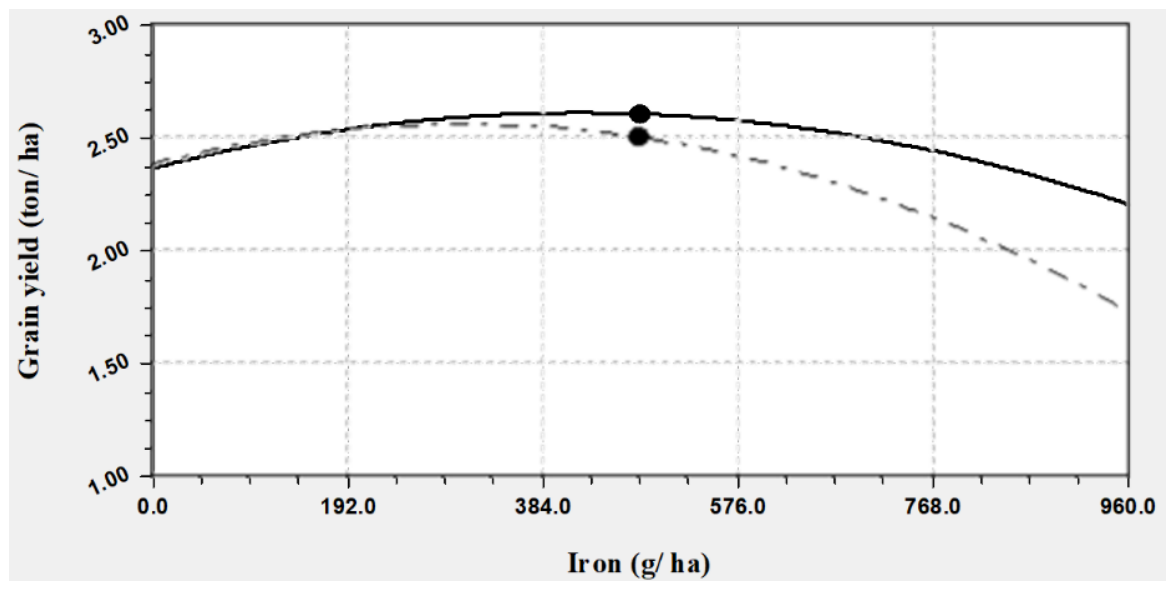

Fig. 1-a. 1- Casino

\footnotetext{
Without humic: $\hat{\mathrm{Y}}=2.37+1.17 * 10^{-3} \mathrm{X}-1.39 * 10^{-6} \mathrm{X}^{2}$ (Optimal: $\left.420.86 \mathrm{~g} / \mathrm{ha}\right)$

- - With humic: $\hat{\mathrm{Y}}=2.30+1.80 * 10^{-3} \mathrm{X}-1.93 * 10^{-6} \mathrm{X}^{2}$ (Optimal: $466.32 \mathrm{~g} / \mathrm{ha}$ )
} 


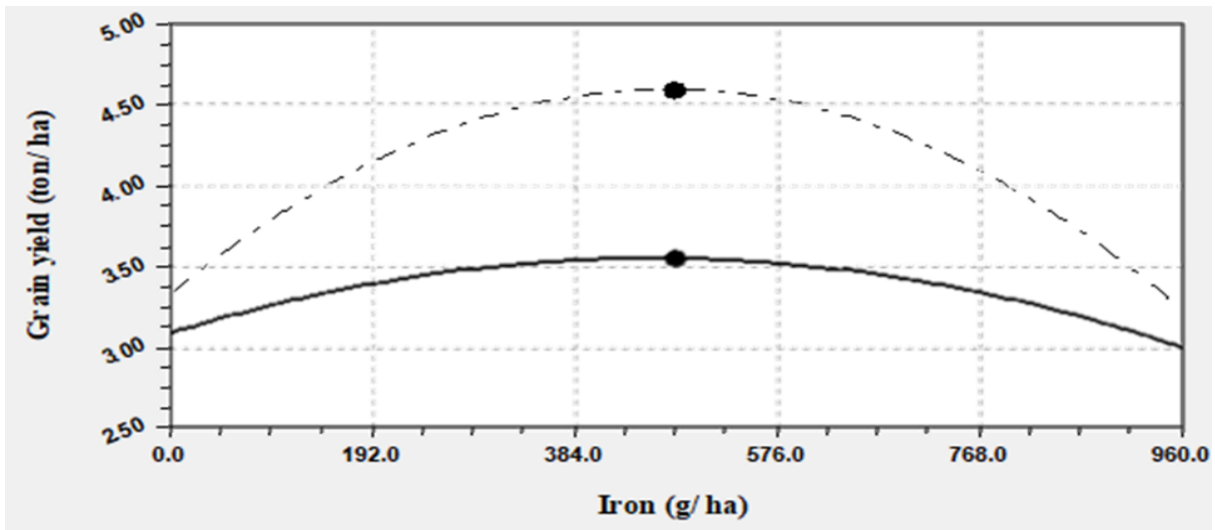

Fig. 1-b. 2- Bani Sweif 6

Without humic: $\hat{\mathrm{Y}}=3.09+2.01 * 10^{-3} \mathrm{X}-2.19 * 10^{-6} \mathrm{X}^{2}$ (Optimal: $458.90 \mathrm{~g} / \mathrm{ha}$ )

- . With humic: $\hat{Y}=3.33+5.38 * 10^{-3} \mathrm{X}-5.70 * 10^{-6} \mathrm{X}^{2}$ (Optimal: $471.92 \mathrm{~g} / \mathrm{ha}$ )

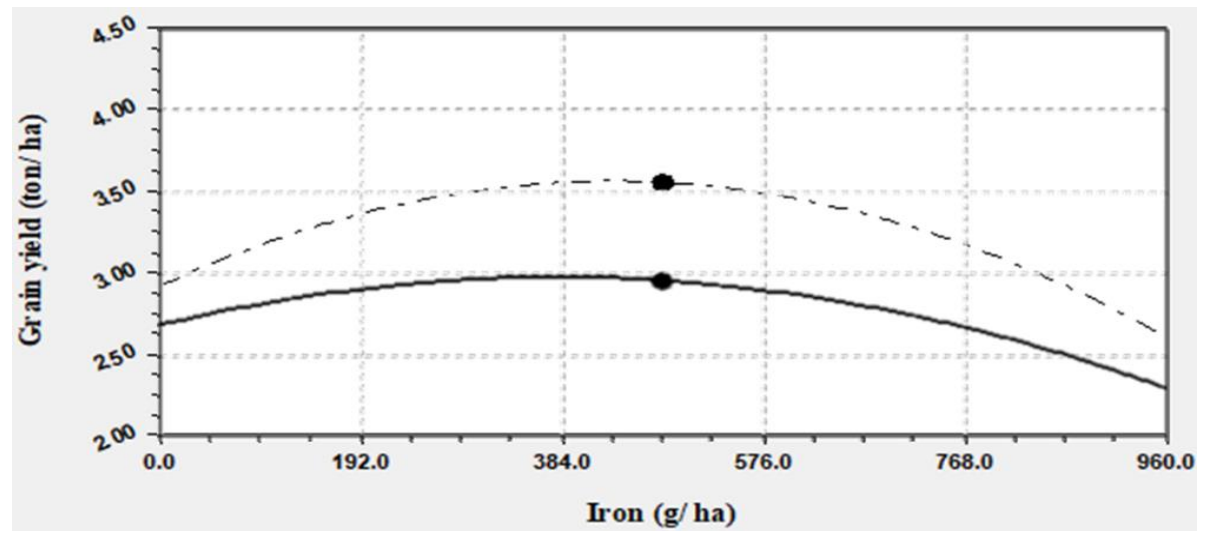

Fig. 1-c. 3- Sohag 3

Without humic: $\hat{Y}=2.69+1.53 * 10-3 \mathrm{X}-2.02 * 10-6 \mathrm{X} 2$ (Optimal: $378.71 \mathrm{~g} / \mathrm{ha}$ )

_ . - With humic: $\hat{Y}=2.92+2.96 * 10-3$ X $-3.43 * 10-6$ X2 (Optimal: $431.48 \mathrm{~g} / \mathrm{ha}$ )

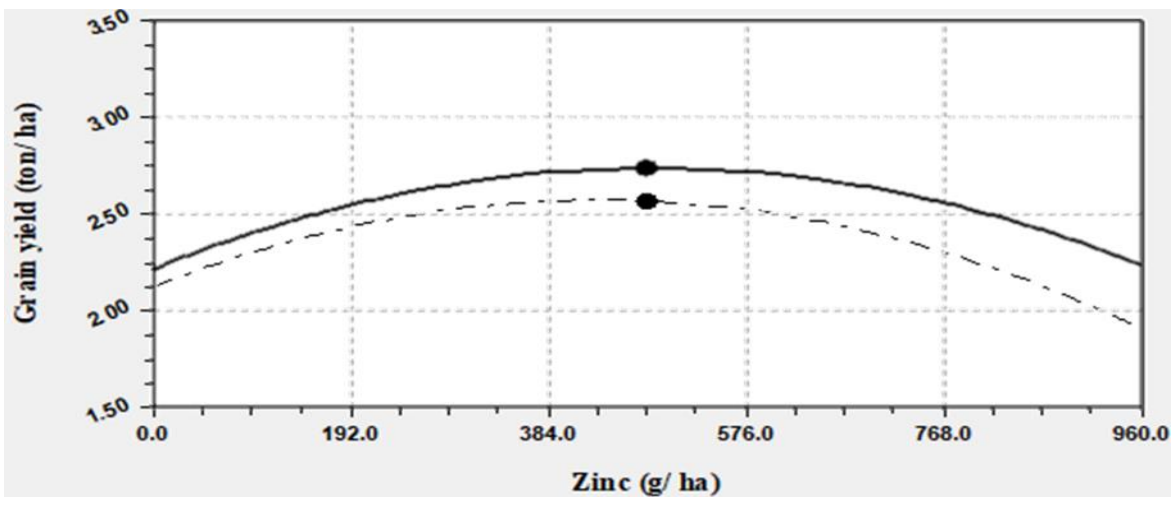

Fig. 2-a. 1- Casino

Without humic: $\hat{\mathrm{Y}}=2.22+2.15 * 10^{-3} \mathrm{X}-2.21 * 10^{-6} \mathrm{X}^{2}$ (Optimal: $486.42 \mathrm{~g} / \mathrm{ha}$ )

_ . _ With humic: $\hat{\mathrm{Y}}=2.13+2.06 * 10^{-3} \mathrm{X}-2.38 * 10^{-6} \mathrm{X}^{2}$ (Optimal: $432.77 \mathrm{~g} / \mathrm{ha}$ ) 


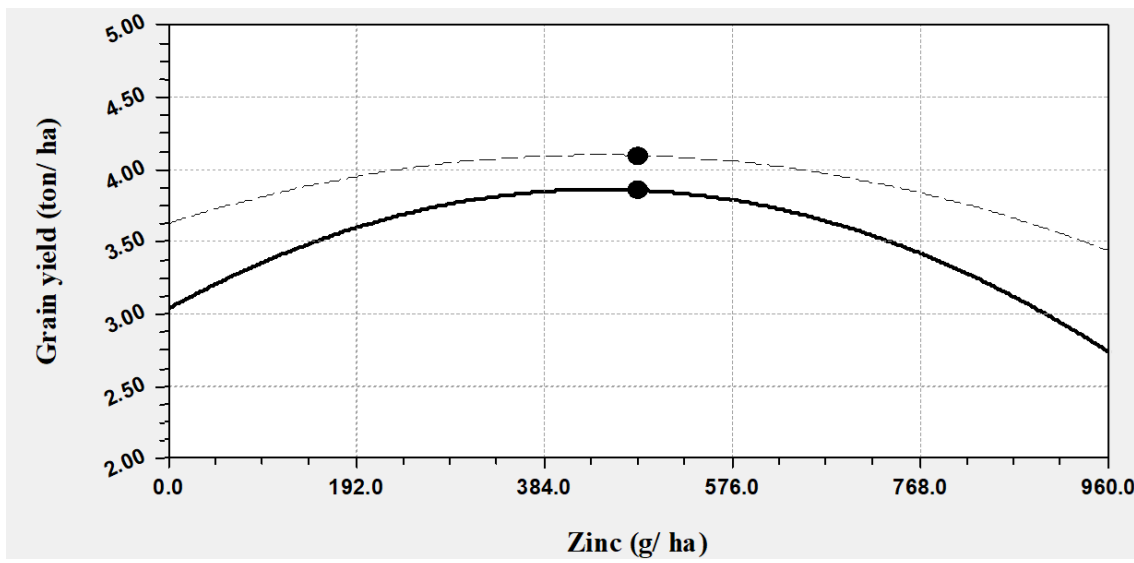

Fig. 2-b. 2- Bani Sweif 6

Without humic: $\hat{\mathrm{Y}}=3.04+3.73 * 10^{-3} \mathrm{X}-4.21 * 10^{-6} \mathrm{X}^{2}$ (Optimal: $\left.442.99 \mathrm{~g} / \mathrm{ha}\right)$

- - With humic: $\hat{\mathrm{Y}}=3.63+2.16 * 10^{-3} \mathrm{X}-2.45 * 10^{-6} \mathrm{X}^{2}$ (Optimal: $\left.440.8 \mathrm{~g} / \mathrm{ha}\right)$

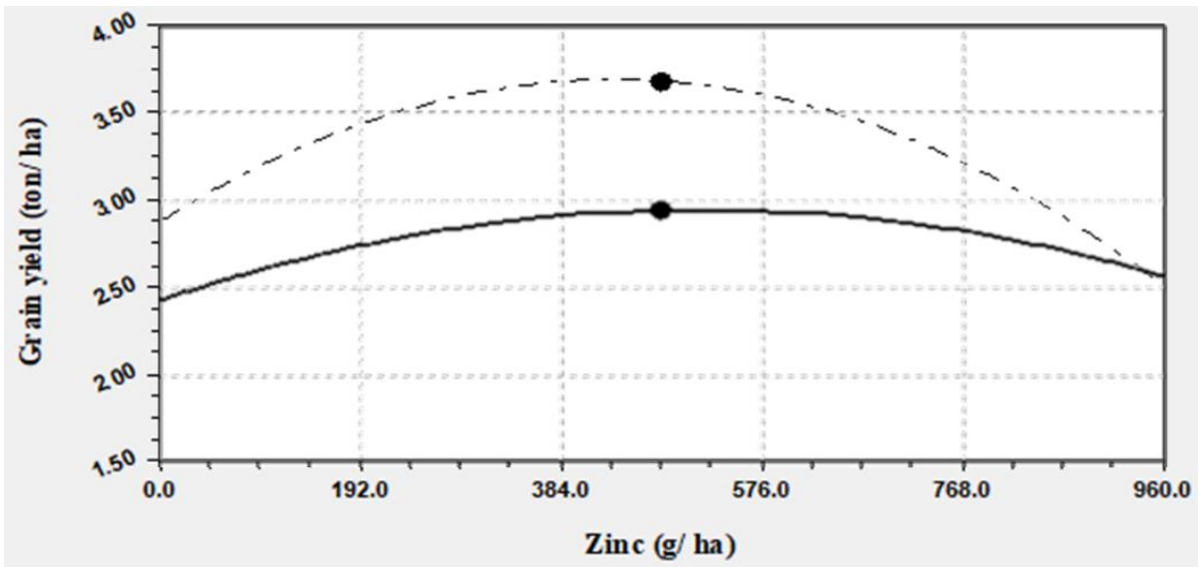

Fig 2-c. 3- Sohag 3

Without humic: $\hat{\mathrm{Y}}=2.43+2.02 * 10^{-3} \mathrm{X}-1.95 * 10^{-6} \mathrm{X}^{2}$ (Optimal: $517.94 \mathrm{~g} / \mathrm{ha}$ )

- - ' With humic: $\hat{\mathrm{Y}}=2.88+3.72 * 10^{-3} \mathrm{X}-4.27 * 10^{-6} \mathrm{X}^{2}$ (Optimal: $435.59 \mathrm{~g} / \mathrm{ha}$ )

\section{CONCLUSIONS}

The present investigation revealed that durum wheat varieties showed a positive response to spraying with both $\mathrm{Fe}$ and $\mathrm{Zn}$ at $480 \mathrm{~g} / \mathrm{ha}$ of each in the form of $\mathrm{FeSO}_{4}$ and $\mathrm{ZnSO}_{4}$. Increasing the level of either/ or both micronutrients caused a significant reduction in yield and yield attributes due to imbalance of the nutritive status of the wheat plant or to indirect effects of excessive levels of the two micronutrients on biochemical process in plants. Durum wheat cultivars showed differential response to HA spraying at 2.4 $\mathrm{kg} / \mathrm{ha}$. That illustrates the need for further experimentation to determine the suitable dose of HA to the different durum wheat cultivars.

\section{REFERENCES}

Al-Erwy, A. S., S. O. Bafeel and A. Al-Toukhy. 2016. Effect of chemical, organic and bio-fertilizers on germination, growth and yield of wheat (Triticum aestivum L.) plants irrigated with sea water. Agric. Biol. J. North Am. 7(3):121-133.

Antoun, Linda. W., Sahar. A. Zakaria and Hanaa. H. Rafla. 2010. Influence of compost, $\mathrm{N}$-mineral and humic acid on yield and chemical composition of wheat plant. J. Soil. Sci. Agric. Eng, Mansoura Univ. 1(11):1131-1143. 
Arif, M., M. Tasneem, F. Bashir, G. Yaseen and A. Anwer. 2017. Evaluation of different levels of potassium and zinc fertilizer on the growth and yield of wheat. Int. J. Biosen. Bioelectron. 3(2):242-246.

Armin, M., S. Akbari and S. Mashhadi. 2014. Effect of time and concentration of nano-Fe foliar application on yield and yield components of wheat. Int. J. Biosci. 4(9):69-75.

Baqir, H. A. and N. H. Zeboon. 2019. Response of some wheat growth traits for foliar spraying with humic and glutamic acid. Iraqi J. Agric. Sci. 50(6):1455-1464.

Broadley, M. R., P. J. White, J. P. Hammond, I. Zelko and A. Lux. 2007. Zinc in plants. New phytologist. 173:677-702.

Delfine, S., R. Tognetti, F. Desiderio and A. Alvino. 2005. Effect of foliar application of $\mathrm{N}$ and humic acids on growth and yield of durum wheat. Agron. Sustion. Dev. 25 (2):183-191.

Dincsoy, M. and F. Sonmez. 2019. The effect of potassium and humic acid application of wheat (Triticum aestivum $\mathrm{L}$. var. Delfii) with some soil properties. J. Plant Nut. 42(20), 2757-2772.

Disante, K. B., D. Fuentes and J. Cortina. 2010. Response to drought of Zn-stressed Quercus suber L. seedlings. Env. Exp. Bot. 70:96-103.

Doroodian, M., Y. Sharghi, A. Alipour and H. Zahedi. 2015. Yield and yield components of wheat as influenced by sowing date and humic acid. Int. J. Nat. Sci. 5(1):8-14.

Finatto, T., A C. de Oliveira, C. Chaparro, L. C. da Maia, D.R. Farias and L. G. Woyann. 2015. Abiotic stress and genome dynamics: specific genes and transposable elements response to iron excess in rice. Rice 8:13. doi: 10.1186/s12284-015-0045-6.

Fogeria, N.K. 2009. The Use of Nutrients in Crop Plants CRc Press, Taylor and Francis group. New York.

Gomaa, M. A., F. I. Radwan, E. E. Kandil and Seham M. A. El-Zweek. 2015. Effect of some macro and micronutrients application methods on productivity and quality of wheat (Triticum aestivum L.). Middle East J. Agric. Res. 4(1):111.

Gomez,K.A.and A.A.Gomez. 1984.Statiscal Procedures for Agricultural Research. 2nded.John Wiley and Sons. New York,USA.

Habib, Maralian. 2009. Effect of foliar application of $\mathrm{Zn}$ and $\mathrm{Fe}$ on wheat yield and quality. Afr. J. Biotech., 8(24):6795-6798.

Habib, Maralian. 2012. Effect of supplementary nutrition with $\mathrm{Fe}, \mathrm{Zn}$ chelates and Urea on wheat quality and quantity. Afr. J. Biotech. 11(11):2661-2665.

Hansch, R. and R. R. Mendel. 2009. Physiological function of mineral micronutrients $(\mathrm{Cu}, \mathrm{Zn}, \mathrm{Mn}, \mathrm{Fe}, \mathrm{Ni}, \mathrm{Mo}, \mathrm{B}, \mathrm{Cl})$. Curr. Opin. Plant Biol. 12:259-266.

Hassanein, M. S., Nabila M. Zaki and Ahmad G. Ahmad. 2019. Effect of zinc foliar application on growth and yield characteristics of two wheat cultivars. Curr. Sci. Int. 8(3):491-498
Hussain, N., M. A. Khan and M. A. Javed. 2005. Effect of foliar application of plant micronutrient mixture on growth and yield of wheat (Triticum aestivum L.). Pak. J. Biol. Sci. 8:1096-1099.

Hyams, D. 2005. Curve Expert Version 1.37. A comprehensive curve fitting package for Windows.

Jalal, A., Sh. Shah, M. C. M. T. Filho, A. Khan, T. Shah, Z. Hussain, M. Younis and M. Ilyas. 2020. Yield and phenological indices of wheat as affected by exogenous fertilization of Zinc and Iron. Rev. Bras. Cienc. Agrar. Recife. 15(1):7730.

Jones, C. A., J. S. Jacobsen and A. Mugaas. 2007. Effect of low-rate commercial humic acid on phosphorus availability, micronutrient uptake, and spring wheat yield. Comm. Soil Sci. Plant Analysis. 38(7-8):921-933.

Kabir, A. H., M. A.Khatun, M. M.Hossain, S. A.Haider, M. F. Alam, and N. K. Paul. 2016. Regulation of phytosiderophore release and antioxidant defense in roots driven by shoot-based auxin signaling confers tolerance to excess iron in wheat. Front. Plant Sci. 7:1684.

Kadkol, G.P. and M. Sissons. 2016. Durum wheat encyclopedia of food grains, 2nd edition. 117-124. Oxford: Academic Press.

Kandil, A. A., A. E. M. Sharief, S. E. Seadh and D. S. k. Altai. 2016. Role of humic acid and amino acids in limiting loss of nitrogen fertilizer and increasing productivity of some wheat cultivars grown under newly reclaimed sandy soil. Int.J. Adv. Res. Biol. Sci. 3(4):123-136.

Kasim, W. A. 2007. Physiological consequences of structural and ultra-structural changes induced by $\mathrm{Zn}$ stress in phaseolus vulgaris. I. Growth and photosynthetic apparatus. Int. J. Bot. 3(1):15-22.

Khaksar, Sara M. and S. Lack. 2019. Evaluation effect of spray micronutrient elements (Mn, $\mathrm{Zn}$ ) on seed yields, its components and protein of wheat (Triticum aestivum L.). J. Crop Nutr. Sci. 5(2):34-46.

Khan, R. U., A. Rahid, M. S. Khan and E. Ozturk. 2010. Impact of humic acid and chemical fertilizer application on growth and grain yield of rainfed wheat (Triticum aestivum L.). Pak. J. Agric. Res. 23(3-4):113-118.

Klute, A. 1986. Methods of Soil Analysis Part 1,2nd ed., Agron. Monor G. ASA and SSSA, Madison. W.I.

Knapowski, T., M. Szczepanek, E. Wilczewski and J. Poberezny. 2015. Response of wheat to seed dressing with humus and foliar potassium fertilization. J. Agric. Sci. Tech. 17:1559-1569.

Kumar, A., S. Dwivedi, R. P. Singh, D. Chakrabarty, S. Mallick and P. K. Trivedi. 2014. Evaluation of amino acid profile in contrasting arsenic accumulating rice genotypes under arsenic stress. Biol. Plant. 58:733-742. doi: 10 .1016/j.jhazmat .2012.06.049.

Li, X., H. Ma, P. Jia, J. Wang, L. Tia, T. Zhang, Y. Tang, H. Chen and X. Wei. 2012. Responses of seedling growth and antioxidant activity to excess iron and copper in (Triticum aestivum L.) Ecotoxicol and Environ. Saf. 86:47-53. 
Lodhi, A., S. Taher, Z. Iqbal, A. Mahmooad, M. Akhtar, T. M. Qureshi, M. Yaqub and A. Naeem. 2013. Characterization of commercial humic acid samples and their impact on growth of fungi and plants. Soil Environment. 32:63-70.

Mackowiak, C. L., P. R. Grossl and B. G. Bugbee. 2001. Beneficial effects of humic acid on micronutrient availability to wheat. Soil Sci. Soc. Am. J. 65(6):17441750.

Manzoor, A., R. A. Khattak and M. Dost. 2014. Humic acid and micronutrient effects on wheat yield and nutrient uptake in salt affected soil. Int. J. Agric. Biol. 16:991-995.

Mekkei, M. E. R. and Eman A. M. A. El Haggan. 2014. Effect of $\mathrm{Cu}, \mathrm{Fe}, \mathrm{Mn}$. $\mathrm{Zn}$ foliar application on productivity and quality of some wheat cultivars (Triticum aestivum L.) J. Agric. food Appl. Sci. 2(9):283-291.

Monjezi, F., F. Vazin and M. H. Zadehdelouei. 2013. Effects of Iron and Zinc spray on yield and yield components of wheat (Triticum aestirum L.) in drought stress. Cercetari Agron in Moldova. 46(1):23-32.

Nadim, M. A., I. U. Awan, M. S. Baloch, E. A. Khan, K. Naveed and M. A. Khan. 2012. Response of wheat (Triticum aestivum L.) to different micronutrients and their application methods. J. Animal Plant Sci. 22(1):113-119.

Page, A.L, R.H. Miller and D.R. Keeney. 1982. Methods of Soil Analysis.2nd Edn. American Society of Agronomy, Madison, WI. USA.

Phlavan-Rad, M. R. and M. Pessarakli. 2009. Response of wheat plants to zinc, iron and manganese applications and uptake and concentration of zinc, iron and Manganese in wheat grains. Com. Soil Sci. Plant Analysis. 40:13221332.
Posmyk, M. M. and R. Kontek. 2009. Antioxidant enzymes activity and phenolic compounds content in red cabbage seedling exposed to copper stress. Ecotoxicol. Environ. Saf. 72:596-602.

Radwan, F. I., M. A. Gomaa, M. A. A. Naser and I. F. Mussa. 2014. Response of some wheat varieties to humic acid, mineral and biofertilization on productivity and quality. Middle-East J. Agric. Res. 3(3):631-637.

Ramzan, Y., M. B. Hafeez, S. Khan, M. Nadeem, S. Rahman, S. Batool and J. Ahmed. 2020. Biofertification with zinc and iron improves the grain quality and yield of wheat crop. Int. J. plant prod. 14:501-510.

Sandalio, L. M., H.C. Dalurzo, M. Gomez, M. C. RomeroPuertas and L. A. Del Rio. 2001. Cadmium-induced changes in the growth and oxidative metabolism of pea plants. J. Exp. Bot. 52:2115-2126.

SAS. 1985. SAS/ STAT. Guide for personal computers. Version 9 edn. SAS end SAS institute, Cary N.C. USA.

Tsonev, T. and F. J. C. Lidon. 2012. Zinc in plants-An overview. Emr. J. Food Agric. 24(4):322-333.

Wu, L., M. Y. Shhadi, G. Gregorio, E. Mathhis, M. Becker and M. Frei. 2014. Genetic and physiological analysis of tolerance to acute iron toxicity in rice. Rice. 7:8. doi:10.1186/s12284-014-0008-3.

Yasin, M. A. T. and E. E. A. El-Sobky. 2017. Impact of humic acid application and nitrogen fertilizer levels on bread wheat productivity under sandy soil conditions. Zagazig, J. Agric. Res. 44(3):887-897. 


\section{الملخص العربي}

\section{تأثثر إضافة حمض الهيوميك والزنكك والحديد علي المحصول ومكوناته في القمح الصلب}

$$
\text { إدريس عمر المهدي- سامي شعبان الطباخ- علي عيسي نوار - محمود حسن عبدالمنعم }
$$

وزن المائة حبة في الموسمين- وقد ادي الرش بحمض فاد الهيوميك لزيادة محصول الحبوب في كل من الصنفين Sohag3, Bani sweif6 للصنف Casino . ن نتيجة الرش بحمض الهيوميك .من ناحية أخري أدي الرش بحمض الهيوميك والحديد والزنك بمعدل •^^ جم /هكتار من كل منهما الي تحقيق اقصي انتاجية من كل من الصنفينSohag3, Bani sweif6 في حين أدي الرش بكل من الحديد والزنك بمعدل •^یـ جم/ هكتار مع عدم الرش بحمض الهيوميك الي تحقيق اقصي محصول للصنف Casino. لذا يمكن التوصية برش صنفي القصح Sohag3, Bani sweif6 وسلفات الحديدوز وسلفات الزنك بمعدل (^^ء جم/هكتار من كل منهما) - في حين يجب إجراء المزيد من الدرسات لتحديد المعدل المناسب من حمض الهيوميك اللازم إضافته للصنف Casino.
نفذت تجربتان حقليتان بالمزرعه البحثية لجامعه

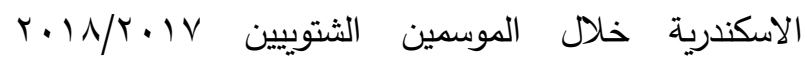

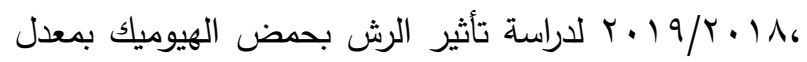
(صفر، ؟. Y كجم /هكتار) وكل من الحديد والزنك بمعدل

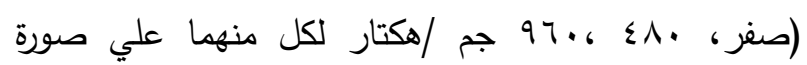

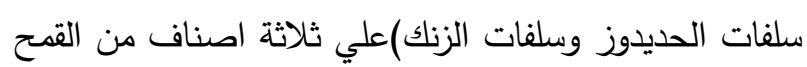
الديورم (Casino, Bani sweif6, Sohag3 )وذلك في تصميم القطع المنشقة مرة واحدة في ثلاث مكررات حيث وزعت

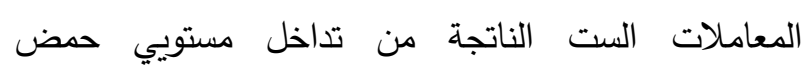
الهيوميك×الاصناف الثثلثة في القطع الرئيسية في حين وزعت المعاملات التسع الناتجة من تداخل المستويات الثلاثة من الزنك×المستويات الثلاثة من الحديد عشوائيا علي القطع منع الفرعية وقد أظهرت النتائج تفوق الصنف Bani sweif6 علي الصنفين الآخرين في محصول الحبوب وجميع مكوناته عدا 